\title{
Budd-Chiari syndrome, a rare complication of multicentric Castleman disease: A case report
}

\author{
KUI SONG ${ }^{1}$ and MIN LI ${ }^{2}$ \\ Departments of ${ }^{1}$ Hematology and ${ }^{2}$ Pharmacy, The First Affiliated Hospital of Jishou University, \\ Jishou, Hunan 416000, P.R. China
}

Received June 30, 2014; Accepted February 2, 2015

DOI: 10.3892/ol.2015.3010

\begin{abstract}
A 39-year-old female presented to The First Affiliated Hospital of Jishou University (Jishou, Hunan) with a fever of unknown origin and progressive abdominal distension. Physical examination revealed generalized lymphadenopathy, multiple non-tender cutaneous nodules, hepatomegaly, splenomegaly and abdominal edema. An axillary lymph node biopsy indicated hyaline vascular type Castleman disease, and color Doppler and computed tomography scans suggested Budd-Chiari syndrome (BCS). Based on the abdominal distension and impairments of the liver and kidneys, an inferior vena cavography and balloon dilatation were performed, confirming the diagnosis of BCS and leading to symptomatic improvement. The patient commenced a combination chemotherapy regimen of cyclophosphamide (0.4 g; days $1-3)$, vindesine (4 mg; day 1) and prednisolone (100 mg; days 1-5), with no melioration of symptoms. Theprubicin was added to suppress the aggravation of the disease on day six of the chemotherapy cycle. The patient exhibited symptomatic remission for one week, however, she subsequently succumbed to intracranial hemorrhage and infections of the lung and intestine due to long-term myelosuppression following chemotherapy. To the best of our knowledge, this is the first report of BCS in a patient with multicentric Castleman disease without human immunodeficiency virus infection.
\end{abstract}

\section{Introduction}

Multicentric Castleman disease (MCD) is a rare, atypical lymphoproliferative disorder, which is characterized by systemic lymphadenopathy and constitutional inflammatory symptoms (1). Due to the rarity of the disease, the incidence of MCD remains unknown (2). The five-year survival rate of

Correspondence to: Dr Min Li, Department of Pharmacy, The First Affiliated Hospital of Jishou University, Shiji Avenue, Jishou, Hunan 416000, P.R. China

E-mail: zs_hematology@163.com

Key words: Multicentric Castleman disease, Budd Chiari syndrome, chemotherapy
MCD ranges between 50 and $77 \%$, following treatment with cytotoxic therapy, glucocorticoids, IFN- $\alpha$, surgical excision or radiotherapy (3). Although MCD is non-neoplastic, without treatment, the prognosis is poor. Infections, multiorgan failure, renal failure and the development of malignancies, including lymphoma (Hodgkin and non-Hodgkin) or Kaposi sarcoma, are common causes of mortality in patients with MCD (4).

Budd-Chiari syndrome (BCS) is an uncommon disease characterized by complete or incomplete hepatic venous and/or intrahepatic inferior vena cava outflow obstruction (5). The majority of cases are idiopathic, although pregnancy, hematological or other diseases, and tumor cell invasion into the hepatic vein or inferior vena cava, are recognized causes of BCS (6).

To the best of our knowledge, an association between BCS and MCD has not been reported previously. The current study reports the case of a female with MCD, who presented with clinical and laboratory features indicative of BCS. Written informed consent was obtained from the patient.

\section{Case report}

A 39-year-old female was admitted to The First Affiliated Hospital of Jishou University (Jishou, China) for one month due to fever combined with progressive abdominal distension, abdominal pain and vomiting. Prior to hospitalization, the patient had experienced an intermittent mild fever $\left(\sim 38.0^{\circ} \mathrm{C}\right)$; intravenous administration of antibiotics and fluid replacement were used to control the fever, with limited efficacy. Upon admission, the patient was febrile with a slight pallor, and exhibited bilateral cervical, axillary and inguinal lymphadenopathy. A systemic examination revealed a uniformly distended abdomen with tense ascites and no palpable mass, and the liver was enlarged. The spleen was palpable $8 \mathrm{~cm}$ below the left costal margin. Mild edema was observed in the lower extremities. Blood analysis revealed a hemoglobin $(\mathrm{Hb})$ level of $102 \mathrm{~g} / 1$, white blood cell (WBC) count of 3.2x10 $/ 1$ (normal range, $4.0-10.0 \times 10^{9} / 1$ ), and platelet (PLT) count of $47 \times 10^{9} / 1$ (normal range, 100-300x10\%/1).Laboratory datashowed elevated serum bilirubin [total bilirubin (TB), $33.0 \mu \mathrm{mol} / 1$ (normal range, 3.4-17.1 $\mu \mathrm{mol} / 1$ ); direct bilirubin (DB), $12.0 \mu \mathrm{mol} / 1$ (normal range, 0-6.8 $\mu \mathrm{mol} / \mathrm{l}$ )] and alkaline phosphatase levels of $231 \mathrm{U} / 1$ (normal range, 40-110 U/1), with normal alanine and aspartate aminotransferase levels. Total serum 

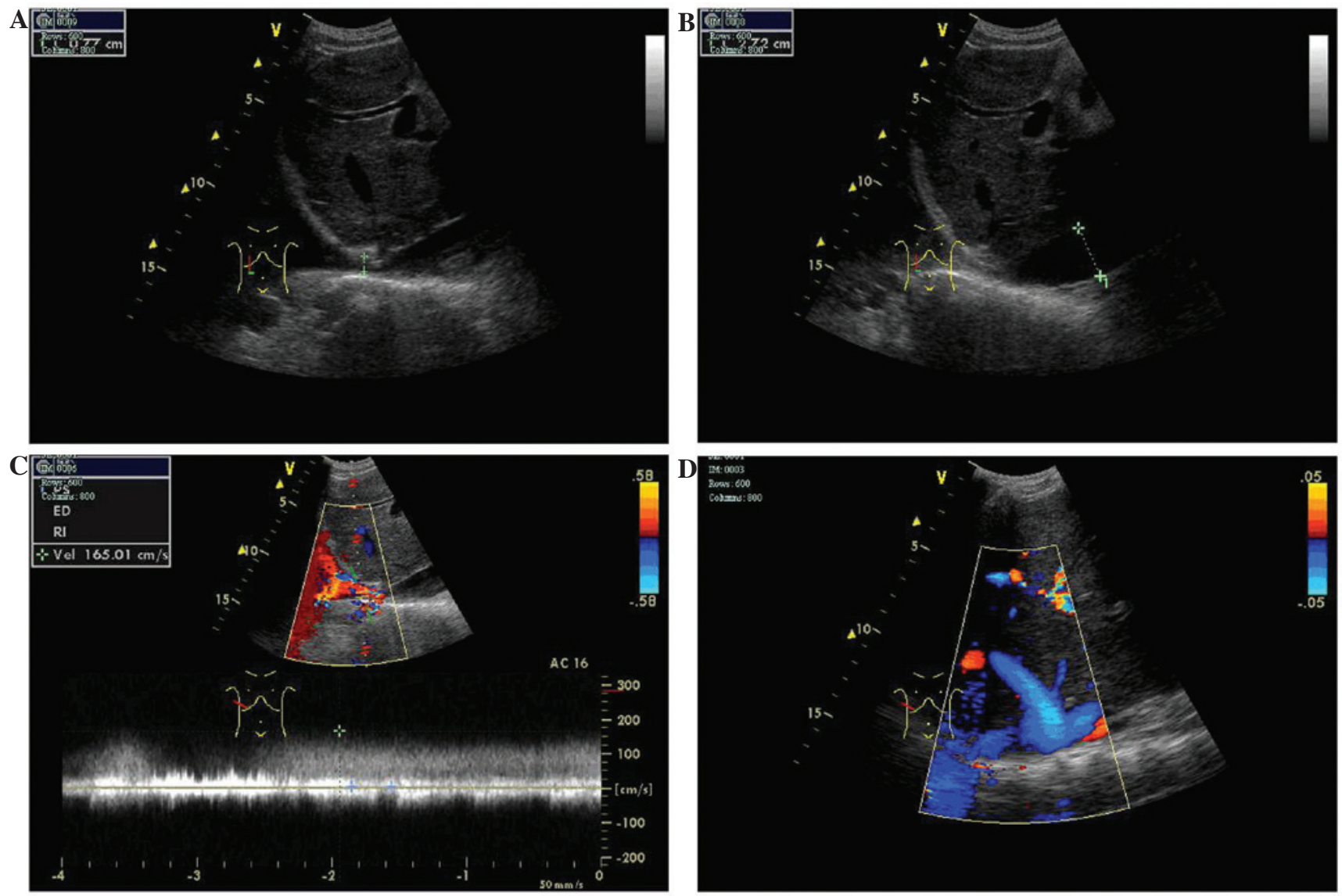

Figure 1. Ultrasound scan of liver revealed hypertrophy, a typical finding in Budd-Chiari syndrome. (A) B mode image indicated a membranous stenosis of the proximal inferior vena cava, with an internal diameter of $0.7 \mathrm{~cm}$ (arrow); (B) B mode image indicated the dilated inferior vena cava distal to the membranous stenosis, with an inside diameter of $2.7 \mathrm{~cm}$ (arrow); (C) CDFI showed a color flow in the stenotic inferior vena cave (arrow), and pulsed-wave tissue Doppler imaging revealed an accelerated centripetal flow to pass by the stenosis, with a flow rate of $1.65 \mathrm{~m} / \mathrm{s}$; (D) CDFI of hepatic vein displayed a typical hepatofugal flow (arrow). CDFI, Color Doppler flow image.

A

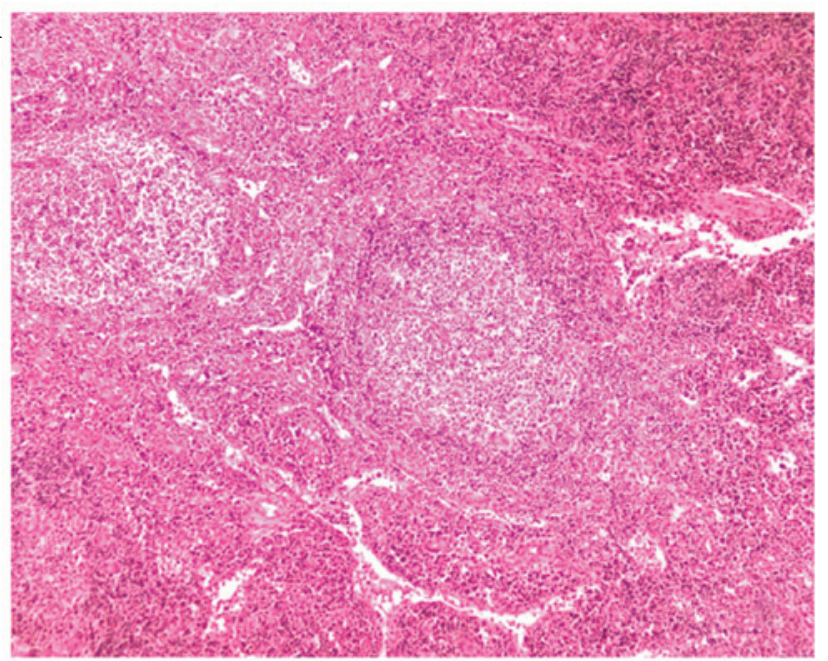

B

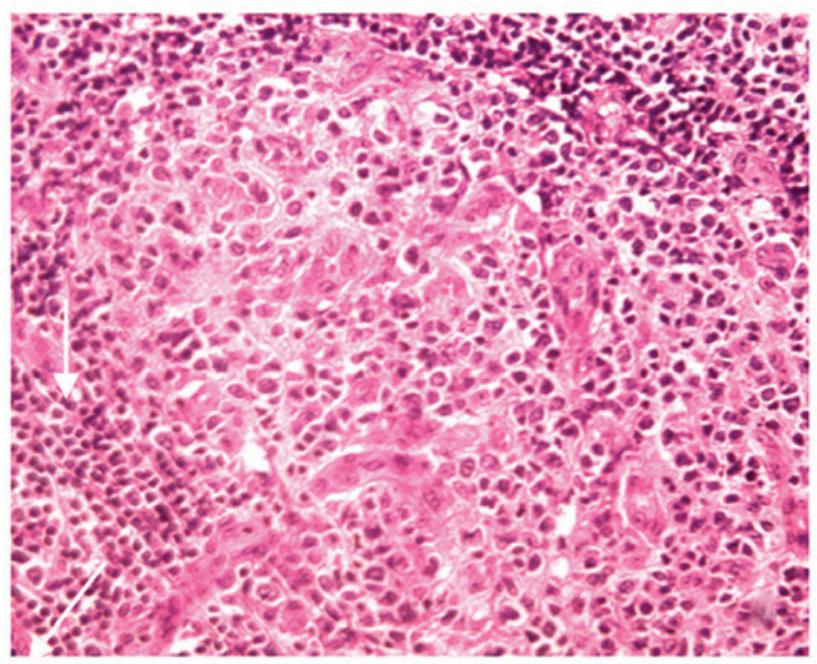

Figure 2. Sections of the right axillary lymph node show lymph follicle hyperplasia. (A) A number of the germinal centers were expanded and surrounded by multilayer small lymphocytes which formed an 'onion-skin' pattern (hematoxylin and eosin staining; magnification, x100). (B) The extensive blood capillary hyperplasia accompanied by hyalinization was distributed in interfollicular areas (hematoxylin and eosin staining; magnification, $\mathrm{x} 400$ ).

protein levels were within the normal range $(62.4 \mathrm{~g} / 1$; normal range, $60.0-80.0 \mathrm{~g} / \mathrm{l}$ ), with albumin of $36.2 \mathrm{~g} / 1$ (normal range, 40.0-55.0 g/l). C-reactive protein levels were elevated, at $72.7 \mathrm{mmol} / \mathrm{l}$ (normal, $<20 \mathrm{mmol} / \mathrm{l}$ ), and lactate dehydrogenase
(LDH) was measured at $270 \mathrm{IU} / 1$ (normal, $<225 \mathrm{IU} / \mathrm{l})$. Tests for human immunodeficiency virus (HIV) were negative. Bone marrow examination revealed megakaryocytosis with insufficient platelet production. An abdominal computed 
tomography scan showed hepatic cirrhosis, an enlarged liver, splenomegaly and splenic hilar varicose veins. Incidentally, multiple enlarged mesenteric and retroperitoneal lymph nodes were observed. Color Doppler studies revealed alterations in the inferior caval vein in the posterior segment of the liver, including distal expansion of the hepatic vein. These findings were consistent with BCS. However, liver and kidney function deteriorated over a period of three weeks. On day 21 of hospitalization, the patient's blood chemistry was as follows: Total protein, $75.5 \mathrm{~g} / 1$; albumin, $26.0 \mathrm{~g} / \mathrm{l}$; alkaline phosphatase, $375 \mathrm{U} / \mathrm{l}$; TB, $81 \mu \mathrm{mol} / \mathrm{l}$; DB, $43 \mu \mathrm{mol} / 1$; urea nitrogen, $24.4 \mathrm{mmol} / \mathrm{l}$; and creatinine, $138 \mu \mathrm{mol} / \mathrm{l}$. Inferior vena cavography and balloon dilatation were successfully performed two days later, resulting in a marked alleviation of abdominal distension. Blood chemistry results indicated favorable response to the surgery. However, the patient's fever was not resolved. A lymph node biopsy revealed hyperplasia, neoplastic transformation of plasma cells and the presence of blood vessels in the lymph nodes, consistent with MCD. At 60 days after admission, a hemogram revealed disease aggravation (WBC, $1.8 \times 10^{9} / 1 ; \mathrm{Hb}, 76 \mathrm{~g} / 1$; PLT, 3x10\% $/ 1$ ). The patient underwent one cycle of combination chemotherapy with cyclophosphamide (0.4 g; days 1-3), vindesine (4 mg; day 1) and prednisolone (100 mg; days 1-5) at 62 days post-admission. However, the fever persisted, with no apparent focus of infection. As the chemotherapy and antibiotic therapy appeared to be ineffective in treating the clinical symptoms, theprubicin (30 mg; days 6-7) was administered in combination with the ongoing chemotherapy regimen. This resulted in symptomatic remission, with alleviated abdominal distension due to the diminution of the liver and spleen, and a normal body temperature. However, myelosuppression occurred for two weeks following chemotherapy, leading to the development of lung and intestinal infections, with high-grade fever and agranulocytosis; this persisted despite treatment with potent antibiotics (0.2 intravenous itraconazole, once a day; 1.0 intravenous imipenem, every $8 \mathrm{~h} ; 0.4$ intravenous teicoplanin, once a day), granulocyte colony-stimulating factor, red blood cell/platelet transfusion and fluid and electrolyte balance was maintained. The patient succumbed to intracranial hemorrhage 74 days after admission.

\section{Discussion}

In comparison to other hematological malignancies, MCD is less frequent and exhibits more malignant behavior, with the involvement of multiple lymph nodes and extranodal sites (2). In the present case, the patient presented with lymphadenopathy, hepatosplenomegaly and systemic manifestations. A lymph node biopsy revealed features of the hyaline vascular type of Castleman disease. Therefore, a diagnosis of multicentric, hyaline vascular type, HIV-negative Castleman disease with BCS manifestations was determined.

Patients with BCS typically present with hepatomegaly, splenomegaly and massive ascites (5). Color Doppler flow image (CDFI) has been commonly utilized as the predominant non-invasive technique for the diagnosis of BCS in recent decades. Compared with surgery, radiological intervention has been reported to be a more effective method for the diagnosis and treatment of BCS, with fewer complication (7). In the current study, a diagnosis of BCS was confirmed by CDFI and inferior vena cavography. Balloon dilatation, performed following inferior vena cavography, resulted in a marked alleviation of BCS manifestations in the short term.

Due to its rarity, and the lack of knowledge with regard to its cause, the optimal therapy for MCD is currently unclear $(2,3)$. The few reported cases were treated using different techniques $(2,3)$, including chemotherapy and radiotherapy, as well as treatment with IL- 6 antibody, glucocorticoids and IFN- $\alpha$, therefore, no standard therapy has been established. In this case, combination chemotherapy was utilized as human leukocyte antigen-matched donors and clinical interleukin- 6 antibody were not available. Combination chemotherapy has previously shown response rates of $\leq 90 \%$ (8), and $\sim 50 \%$ of patients exhibited sustained complete responses with four-drug combination chemotherapy $(9,10)$. In the current case, the patient succumbed to intracranial bleeding following chemotherapy with cyclophosphamide, doxorubicin, vincristine and prednisone. However, the unfavorable outcome may have been related to BCS and the patient's poorer physical condition, and may not be indicative of limited efficacy of the combination chemotherapy.

To the best of our knowledge, BCS has not previously been reported in association with MCD. In the current case, the membranous stenosis of the inferior vena cava was considered to be the primary cause. However, the role of MCD in the development of the stenosis, which resulted in BCS, remains unclear. Various etiologies including idiopathic membranes, neoplasia, infection, trauma and total parenteral nutrition have been reported (11). Eren et al reported a case of BCS secondary to adrenal tumor compression (12). In the present case, the long-term compression of the inferior vena cava due to hepatomegaly may have contributed to the development of the condition. Another possible cause is the neoplastic transformation caused by MCD, which may invade and alter the vessel wall structure of the inferior vena cava (12). However, membranous stenosis or obstruction of inferior vena cava were considered to be the predominant causes.

MCD and BCS are extremely rare, particularly in combination; to the best of our knowledge, this is the first reported case of MCD associated with BCS. Combination chemotherapy was used to treat the conditions, resulting in an unfavorable outcome. However, limited data are currently available with regard to these diseases, and further reports of similar cases may lead to the development of more effective diagnostic tools and standard therapies.

\section{References}

1. Kojima M,Nakamura N, Tsukamoto N, et al: Clinical implications of idiopathic multicentric castleman disease among Japanese: a report of 28 cases. Int J Surg Pathol 16: 391-398, 2008.

2. Robinson D Jr, Reynolds M, Casper C, et al: Clinical epidemiology and treatment patterns of patients with multicentric Castleman disease: results from two US treatment centres. Br J Haematol 165: 39-48, 2014.

3. Seo S, Yoo C, Yoon DH, et al: Clinical features and outcomes in patients with human immunodeficiency virus-negative, multicentric Castleman's disease: a single medical center experience. Blood Res 49: 253-258, 2014.

4. Enomoto K, Nakamichi I, Hamada K, et al: Unicentric and multicentric Castleman's disease. Br J Radiol 80: e24-e26, 2007.

5. Nozari N, Vossoghinia H, Malekzadeh F, et al: Long-term Outcome of Budd-Chiari Syndrome: A Single Center Experience. Middle East J Dig Dis 5: 146-150, 2013. 
6. Bargalló X, Gilabert R, Nicolau C, et al: Sonography of Budd-Chiari syndrome. AJR Am J Roentgenol 187: W33-W41, 2006.

7. Hoekstra J and Janssen HL: Vascular liver disorders (I): diagnosis, treatment and prognosis of Budd-Chiari syndrome. Neth J Med 66: 334-339, 2008

8. Miralles GD, O'Fallon JR and Talley NJ: Plasma-cell dyscrasia with polyneuropathy. The spectrum of POEMS syndrome. N Engl J Med 327: 1919-1923, 1992.

9. Chronowski GM, Ha CS, Wilder RB, et al: Treatment of unicentric and multicentric Castleman disease and the role of radiotherapy. Cancer 92: 670-676, 2001.

10. Powles T, Stebbing J, Montoto S, et al: Rituximab as retreatment for rituximab pretreated HIV-associated multicentric Castleman disease. Blood 110: 4132-4133, 2007.
11. Qi X, Wu F, Ren W, et al: Thrombotic risk factors in Chinese Budd-Chiari syndrome patients. An observational study with a systematic review of the literature. Thromb Haemost 109: 878-884, 2013.

12. Eren $\mathrm{S}$ and Akdağ R: Rare complication of adrenal neuroblastoma: Budd-Chiari syndrome. Pediatr Int 45: 737-739, 2003. 